\title{
9th NCI-EORTC SYMPOSIUM ON NEW DRUGS IN CANCER THERAPY
}

March 12-15,1996

Amsterdam, The Netherlands

The series of NCl-EORTC Symposia on New drugs in Cancer Therapy has been receiving increasing appreciation and recognition. This bi-annual "Amsterdam New Drug Meeting" is now perceived by many as one of the most exciting conferences in the field of new anticancer drug development. It is therefore a pleasure to announce that preparations for the next NCI-EORTC Symposium have been initiated. The Organizing Committee invites you to reserve March 12-15, 1996 for the 9th NCI EORTC Symposium on New Drugs in Cancer Therapy.

International organizing committee Symposium president: H.M. Pinedo

J.P. Armand

B. A. Chabner

T. A. Connors

G. A. Curt

M. D'Incalci

M. A. Friedman

H. H. Hansen

R. E. C. Henrar

C. K. van Kalken

M. W. Lobbezoo

D. L. Longo

J. G. McVie

D. Shoemaker

D. Parkinson

J. B. Vermorken

J. Verweij

O. C. Yoder

\section{Scientific advisory board}

C. J. Allegra

S. G. Arbuck

J. H. Beijnen

J. R. Bertino

B. Chevallier

A. H. Calvert

M. Christian

R. C. Donehower

Ch. Dittrich

J. Double

G. Eisenbrand

E. A. Eisenhauer

H. H. Fiebig

G. Giaccone

C. K. Grieshaber

K. K. Harrap

H. R. Hendriks

J. Mendelsohn

C. E. Myers

M. Ogawa

I. Pastan

M. Potmesil

E. A. Sausville

S. Seeber

F. Valeriote

D. D. Von Hoff

J. Wagstaff

R. E. Wittes

H. Zwierzina
Amsterdam, The Netherlands Villejuif, France Bethesda, U.S.A. Carshalton, U.K. Bethesda, U.S.A. Milano, Italy Bethesda, U.S.A. Copenhagen, Denmark Amsterdam, The Netherlands Amsterdam, The Netherlands Amsterdam, The Netherlands Bethesda, U.S.A. London, United Kingdom Bethesda, U.S.A. Bethesda, U.S.A.

Amsterdam, The Netherlands Rotterdam, The Netherlands Brussels, Belgium

Bethesda, U.S.A. Bethesda. U.S.A.

Amsterdam, The Netherlands New York, U.S.A. Rouen, France Newcastle, U.K. Bethesda, U.S.A. Baltimore, U.S.A. Vienna, Austria Bradford, U.K.

Kaiserslautern, Germany Kingston, Canada Freiburg, Germany

Amsterdam, The Netherlands Rockville, U.S.A. Sutton, U.K.

Amsterdam, The Netherlands New York, U.S.A. Richmond, U.S.A. Tokyo, Japan Bethesda, U.S.A. New York, U.S.A. Bethesda, U.S.A. Essen, Germany Detroit, U.S.A. San Antonio, U.S.A. Amsterdam, The Netherlands Bethesda, U.S.A. Innsbruck, Austria 